\title{
Statistical and dynamical aspects in the decay of hot neutron-rich nuclei
}

\author{
M. Veselský ${ }^{1}$, A.L. Keksis ${ }^{2}$, G.A. Souliotis ${ }^{2}$, K. Wang ${ }^{3}$, \\ E. Bell ${ }^{2}$, D.V. Shetty ${ }^{2}$, M. Jandel ${ }^{2}$, S.J. Yennello², Y.G. $\mathrm{Ma}^{3}$ \\ ${ }^{1}$ Institute of Physics, Slovak Academy of Sciences, Bratislava, Slovakia \\ ${ }^{2}$ Cyclotron Institute, Texas A\&M University, College Station, USA \\ ${ }^{3}$ Shanghai Institute of Applied Physics, Shanghai, China
}

\begin{abstract}
A signal of isospin-asymmetric phase transition in the evolution of the chemical potential was observed in hot quasi-projectiles produced in the reactions ${ }^{40,48} \mathrm{Ca}+{ }^{27} \mathrm{Al}$ confirming an analogous observation in the lighter quasi-projectiles observed in the reaction ${ }^{28} \mathrm{Si}+{ }^{112,124} \mathrm{Sn}$ [1. With the increasing mass, the properties of hot quasi-projectiles become increasingly influenced by secondary emission. Thermodynamical observables exhibit no sensitivity to a different number of missing neutrons in the two reactions ${ }^{40,48} \mathrm{Ca}+{ }^{27} \mathrm{Al}$ and provide a signal of dynamical emission of neutrons, which can be related to a very neutron-rich low-density region ( neck ) between the projectile and target.
\end{abstract}

\section{Introduction}

The isotopic composition of nuclear reaction products provides an important information on reaction dynamics and on possible occurrence of a phase transition in the isospin asymmetric nuclear matter [2, 3], which is supposed to separate into a symmetric dense phase and an asymmetric dilute phase. It has been discussed in the literature [4] to what extent is such a phase transition generated by fluctuations of density or concentration, typically 
suggesting a coupling of both instability modes. The $\mathrm{N} / \mathrm{Z}$ (neutron to proton ratio) degree of freedom and its equilibration was studied experimentally in detailed measurements of the isotopic distributions of emitted fragments [5, 6, 7, 8, 9]. Isotopically resolved data in the region of $\mathrm{Z}=2-8$ revealed systematic trends, which were, however, substantially affected by the decay of the excited primary fragments.

In order to investigate thermodynamical properties of the hot multifragmentation source, it has to be properly characterized in terms of the production mechanism and the level of equilibration. The fragment data from the reactions ${ }^{28} \mathrm{Si}+{ }^{112,124} \mathrm{Sn}$ at projectile energy of 30 and $50 \mathrm{MeV} /$ nucleon [1, 10, 11, 12, 13] provided full information (with the exception of emitted neutrons) on the decay of thermally equilibrated hot quasi-projectiles with known mass $(A=20-30)$, charge, velocity and excitation energy. An excellent description of both the dynamical properties of the reconstructed quasi-projectile such as velocity, excitation energy and isospin-asymmetry as well as the fragment observables such as multiplicity, charge and isotope distributions was obtained [10] using the model of deep-inelastic transfer (DIT) [14] for the early stage of collisions, and the statistical multifragmentation model (SMM) [15] for de-excitation. The contribution from non-equilibrium processes such as pre-equilibrium emission was shown to be weak [10]. The model calculation shown that the average number of neutrons not detected in the experiment was between one and two per event. Therefore, no sig-

nificant distortion of the results can be expected. The observed trends of thermodynamical observables provided several correlated signals of isospinasymmetric liquid-gas phase transition [1, 13, in particular a unique signal seen in the evolution of the isovector chemical potential [1].

The present work is an extension of these studies, with the aim to investigate the effect of the mass of hot nuclei on observed thermodynamical properties and related signals of isospin-asymmetric liquid-gas phase transition.

\section{Experiment}

The experiment was performed at the Cyclotron Institute of Texas A\&M University, using $45 \mathrm{MeV} /$ nucleon ${ }^{40,48} \mathrm{Ca}$ beams delivered by the K500 superconducting cyclotron impinging on $\mathrm{Al}\left(2.2 \mathrm{mg} / \mathrm{cm}^{2}\right)$ target. The multidetector array FAUST [16] was employed covering forward angles between 2 
and $35^{\circ}$, where fragments originating from the projectile-like source can be expected due to beam velocity. FAUST consists of 68 charged particle telescopes arranged in five rings. Each particle telescope consists of a $300 \mu \mathrm{m}$ thick silicon detector followed by a $3 \mathrm{~cm}$ thick $\mathrm{CsI}(\mathrm{Tl})$ crystal. The angular ranges covered by the rings were chosen in order to distribute the multiplicity of detected particles evenly, thus avoiding cases where one telescope is hit simultaneously by multiple particles. In the experiment, the mass and atomic number of the detected charged particles were identified up to $\mathrm{Z}=8$, for the detectors in the forward rings.

The method of isotope identification uses a particle telescope technique in which the isotopes are resolved in the two-dimensional $\Delta$ E-E spectra. We used a method [17] which enables to perform the isotope identification and energy calibrations simultaneously using a minimization procedure. In the experimental spectra, the lines for three known isotopes (typically ${ }^{1} \mathrm{H},{ }^{4} \mathrm{He}$, ${ }^{7} \mathrm{Be}$ ) are assigned and energy calibration is performed by the minimization procedure where these lines are fitted to corresponding calculated energy losses. The calibration coefficients are thus obtained as optimum values of the minimization parameters. The silicon detectors were calibrated using an alpha-source while the empirical formula of Tassan-Got [18] was used for the energy calibration of the $\mathrm{CsI}(\mathrm{Tl})$ crystals.

\section{Analysis}

Using the calibration and identification procedures described above, it was possible to identify the charged particles and to determine their energies on an event-by-event basis. For the subset of events where all detected charged particles were identified, it was possible to reconstruct the mass, charge and excitation energy of the composite system, in the same way as in the previous work [10]. The analysis was performed on the subset of events with total charge larger than that of the projectile $(Z \geq 21)$, thus selecting events where the incomplete fusion, occurring in mid-central collisions, is the dominant contributing reaction mechanism.

\section{Statistical decay of the hot source with mass $40-50$}

One of the goals of the present work was to verify the behavior observed in the reaction ${ }^{28} \mathrm{Si}+{ }^{112,124} \mathrm{Sn}$. Of special interest was the verification of the 
signal of the liquid-gas phase transition, obtained using isoscaling analysis in our previous work [1]. Isoscaling [19] is observed when the ratio of isotopic yields from two reactions with a different isospin asymmetry exhibits an exponential dependence on the fragment isospin asymmetry

$$
R_{21}(N, Z)=Y_{2}(N, Z) / Y_{1}(N, Z) \simeq C \exp \left(\beta^{\prime}(N-Z)\right),
$$

where the parameter $\beta^{\prime}$ can be related, in the grand-canonical limit, to the isovector component of the free nucleon chemical potential, since $\beta^{\prime}=\Delta\left(\mu_{n}-\right.$ $\left.\mu_{p}\right) / 2 \mathrm{~T}$.

The fragment data obtained in the reactions ${ }^{28} \mathrm{Si}+{ }^{112,124} \mathrm{Sn}$ at projectile energy 30 and $50 \mathrm{MeV} /$ nucleon [1, 10, 11, 12] provided full information (with the exception of emitted neutrons) on the decay of thermally equilibrated hot quasi-projectiles with known mass $(A=20-30)$, charge, velocity and excitation energy. A simulation employing the model of deep-inelastic transfer (DIT) [14] for the early stage of collisions and the statistical multifragmentation model (SMM) [15] for de-excitation allowed excellent description of both the dynamical properties of the reconstructed quasi-projectile such as velocity, excitation energy and isospin-asymmetry and the fragment observables such as multiplicity, charge and isotope distributions [10]. Thermodynamical properties of the quasi-projectiles undergoing statistical multifragmentation [1, 10, 11, 12] were investigated in order to reveal possible signals of the phase transition. The difference of isovector chemical potentials in the two reactions $\Delta\left(\mu_{n}-\mu_{p}\right)$ was estimated using isoscaling analysis. A turning-point in the trend of the observable $\beta^{\prime} T$ was observed at excitation energy of about 4 $\mathrm{MeV} /$ nucleon. Such a behavior can be understood as a signal of the onset of chemical separation into the dense isospin symmetric and the dilute isospin asymmetric phases which reverts the decreasing trend of the free nucleon chemical potential consistent with expansion of the homogeneous system. Above the turning-point, the temperature determined using the fragment yield ratios agreed well with the kinematic temperature of protons, which can be identified as the remnants of the dilute phase (a nucleon gas). Such agreement between the two methods of thermometry demonstrates that quantitatively, the grand-canonical approach describes the properties of hot nuclei undergoing multifragmentation very well. Besides isoscaling, another grandcanonical scaling was observed since the isobaric yield ratio $\mathrm{Y}\left({ }^{3} \mathrm{H}\right) / \mathrm{Y}\left({ }^{3} \mathrm{He}\right)$ exhibited an exponential dependence on the quasiprojectile $\mathrm{N} / \mathrm{Z}$ ratio. The experimental data from reactions ${ }^{28} \mathrm{Si}+{ }^{112,124} \mathrm{Sn}$ at projectile energy 30 and 
$50 \mathrm{MeV} /$ nucleon are thus well understood in terms of a reaction mechanism, where the projectile and target nuclei form a di-nuclear configuration, exchange a considerable amount of nucleons, and after the re-separation reach thermal equilibrium at different temperatures. The highly excited quasiprojectile continues to expand up to the spinodal contour.
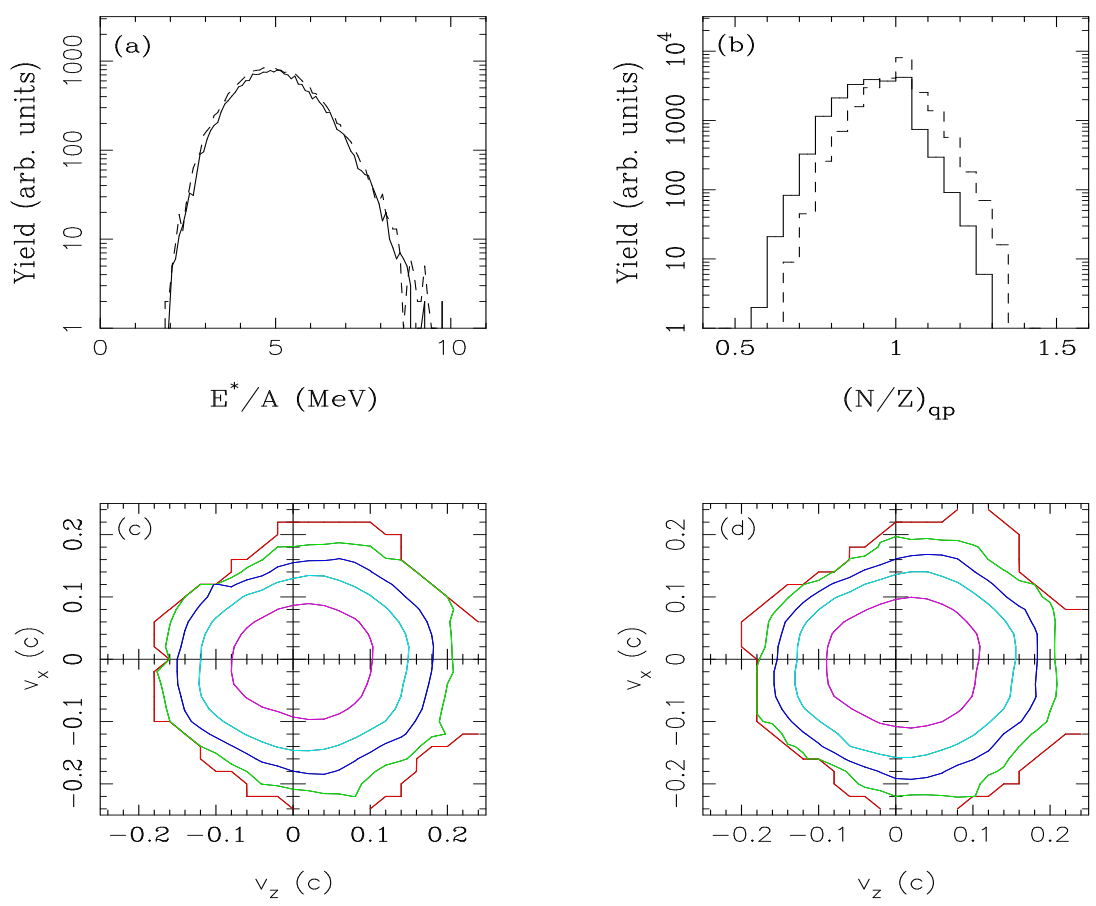

Figure 1: (a) Excitation energy distributions of the reconstructed quasi-projectiles in the reactions ${ }^{40,48} \mathrm{Ca}+{ }^{27} \mathrm{Al}$ at $45 \mathrm{MeV} /$ nucleon (solid line for ${ }^{40} \mathrm{Ca}$ and dashed line for ${ }^{48} \mathrm{Ca}$ ), (b) N/Z-distributions of the reconstructed quasi-projectiles (solid line for ${ }^{40} \mathrm{Ca}$ and dashed line for ${ }^{48} \mathrm{Ca}$ ), (c) Velocity-plot of the light charged particles in the reaction ${ }^{40} \mathrm{Ca}$ $+{ }^{27} \mathrm{Al}$ at $45 \mathrm{MeV} /$ nucleon, (d) Velocity-plot of the light charged particles in the reaction ${ }^{48} \mathrm{Ca}+{ }^{27} \mathrm{Al}$ at $45 \mathrm{MeV} /$ nucleon.

As a first step of the analysis, the characteristics of the projectile-like nuclei, observed at forward angles, were reconstructed on an event-by-event basis. The results for the fully resolved quasiprojectiles with $\mathrm{Z} \geq 21$, which are expected to originate dominantly from incomplete fusion reactions at midcentral impact parameters, are shown in Fig. 1. The excitation energy distributions of the reconstructed quasi-projectiles in the reactions ${ }^{40,48} \mathrm{Ca}+{ }^{27} \mathrm{Al}$ at $45 \mathrm{MeV} /$ nucleon (see Fig. 1 $\mathrm{a}$ ) are practically identical, what is somewhat 

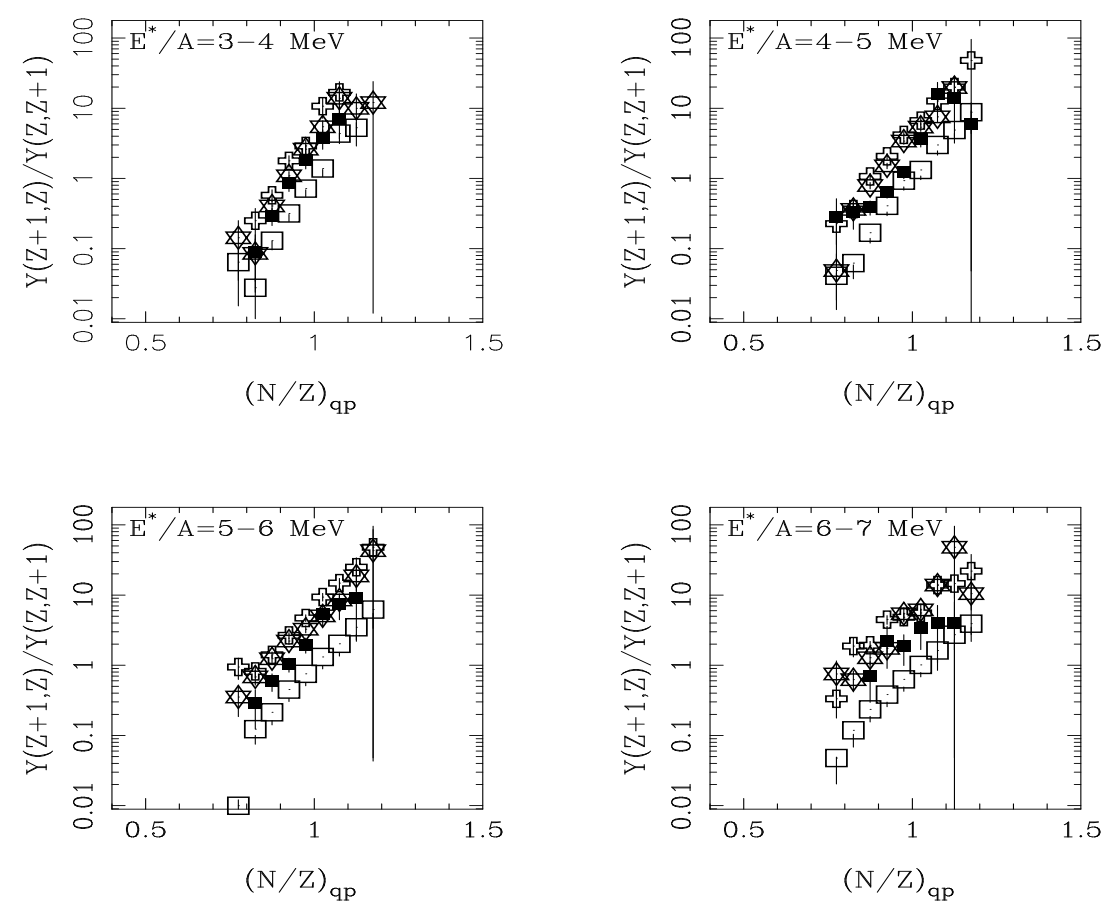

Figure 2: Yield ratios of mirror nuclei $\left({ }^{3} \mathrm{H} /{ }^{3} \mathrm{He}\right.$ - open squares, ${ }^{7} \mathrm{Li} /{ }^{7} \mathrm{Be}$ - thick crosses, ${ }^{11} \mathrm{~B} /{ }^{11} \mathrm{C}$ - stars, ${ }^{15} \mathrm{~N} /{ }^{15} \mathrm{O}$ - solid squares ) observed in the reaction ${ }^{48} \mathrm{Ca}+{ }^{27} \mathrm{Al}$ at 45 $\mathrm{MeV} /$ nucleon plotted for four excitation energy bins as a function of $\mathrm{N} / \mathrm{Z}$ of the reconstructed projectile-like nuclei.

surprising when taking into account that the number of missing (undetected) neutrons may differ considerably. A possible uncertainty in the evolution of neutrons can be documented also by the observed N/Z-distributions (see Fig. 1b) which reflect initial N/Z-difference of the two projectile nuclei (amounting to 0.48 ) only partially, since the mean values (centroids) in the reactions ${ }^{40,48} \mathrm{Ca}+{ }^{27} \mathrm{Al}$ are $\mathrm{N} / \mathrm{Z}=0.93$ and 1.01 , respectively. The velocity-plots of the light charged particles in the quasi-projectile frame in these two reactions are shown in Fig. 1 (for ${ }^{40} \mathrm{Ca}$ on panel $1 \mathrm{k}$ and for ${ }^{48} \mathrm{Ca}$ on panel $1 \mathrm{~d}$ ). Practically isotropic emission is observed which again implies the statistical multifragmentation of the hot projectile-like nucleus. A slight suppression at the backward hemisphere is caused by the combined effect of limited angular coverage and energy thresholds of the experimental device. Using the observed characteristics of the quasi-projectile, the centrality of the observed 
data was estimated using the simulation, successful in describing properties of the hot multifragmenting sources formed in violent collisions in many reactions in the Fermi energy domain [20, 21]. Comparison of the properties of the hot quasiprojectile source leads to conclusion that incomplete fusion collisions at mid-central impact parameters contribute dominantly, mainly due to narrow angular acceptance of the experimental setup around the beam direction for the reconstructed quasi-projectiles with $\mathrm{Z} \geq 21$. Similar selectivity was experimentally observed in heavy residue data in the reaction ${ }^{124} \mathrm{Sn}+{ }^{27} \mathrm{Al}$ [21].
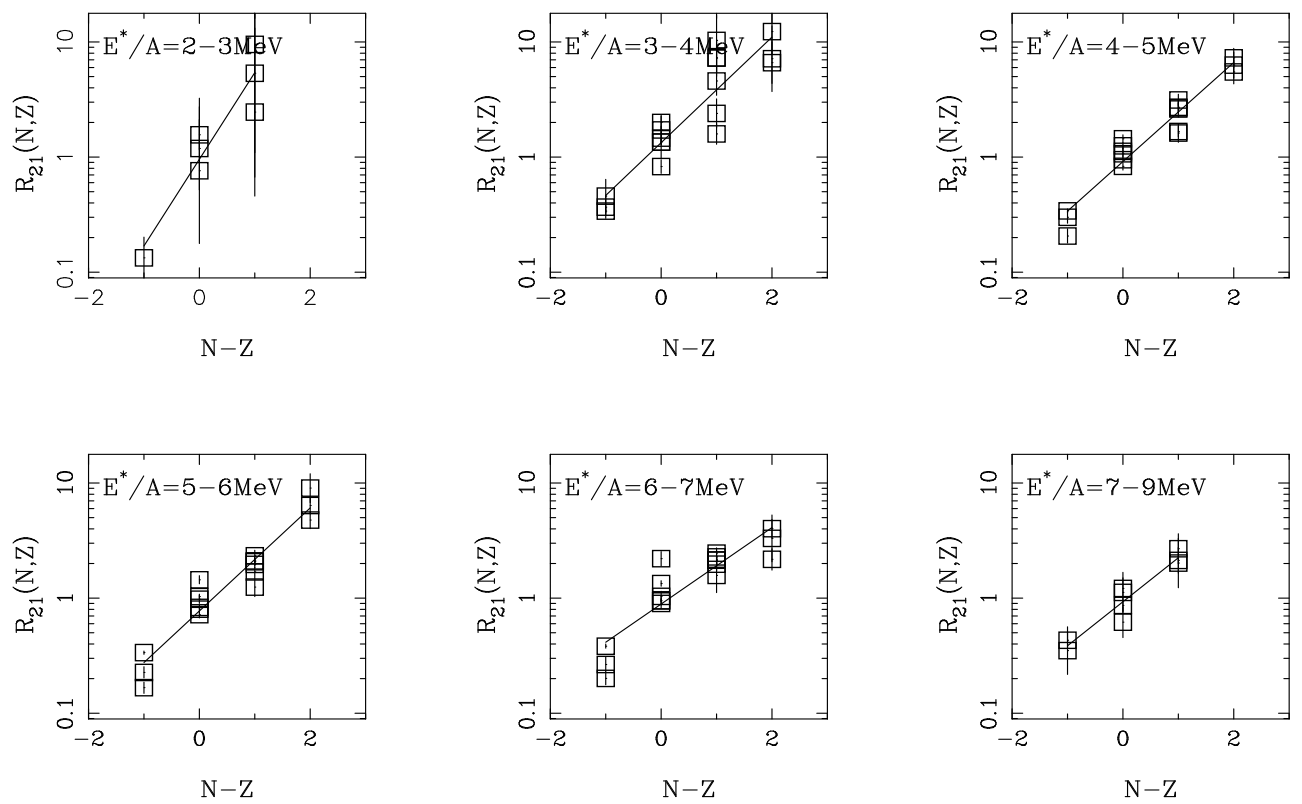

Figure 3: Isoscaling plots, constructed for the pair of reactions ${ }^{40,48} \mathrm{Ca}+{ }^{27} \mathrm{Al}$ at $45 \mathrm{MeV} /$ nucleon using the identified fragments with $\mathrm{Z}=1-8$ forming the fully resolved quasiprojectiles with $\mathrm{Z} \geq 21$ in six excitation energy bins.

Statistical emission in the projectile-like frame is indicated also in Fig. 2. In a similar way as in the reactions ${ }^{28} \mathrm{Si}+{ }^{112,124} \mathrm{Sn}$, where the isobaric ratio ${ }^{3} \mathrm{H} /{ }^{3} \mathrm{He}$ exhibited an exponential (grand-canonical) scaling with the quasiprojectile $\mathrm{N} / \mathrm{Z}$, several yield ratios of mirror nuclei measured in the reaction ${ }^{48} \mathrm{Ca}+{ }^{27} \mathrm{Al}$ at $45 \mathrm{MeV} /$ nucleon are plotted as functions of the $\mathrm{N} / \mathrm{Z}$ of the reconstructed projectile-like nuclei. Again the exponential scaling is observed, with identical slopes for all ratios within each of the excitation 
energy bins. The slope decreases with increasing excitation energy in an analogous way as in the reactions ${ }^{28} \mathrm{Si}+{ }^{112,124} \mathrm{Sn}[12$. The observed grandcanonical scaling provides an evidence that statistical multifragmentation is a dominant mode of de-excitation also in the present case. It is also remarkable to note that essentially no effect of missing neutrons on the observed slope can be seen in this neutron-rich system with a significant number of missing neutrons.

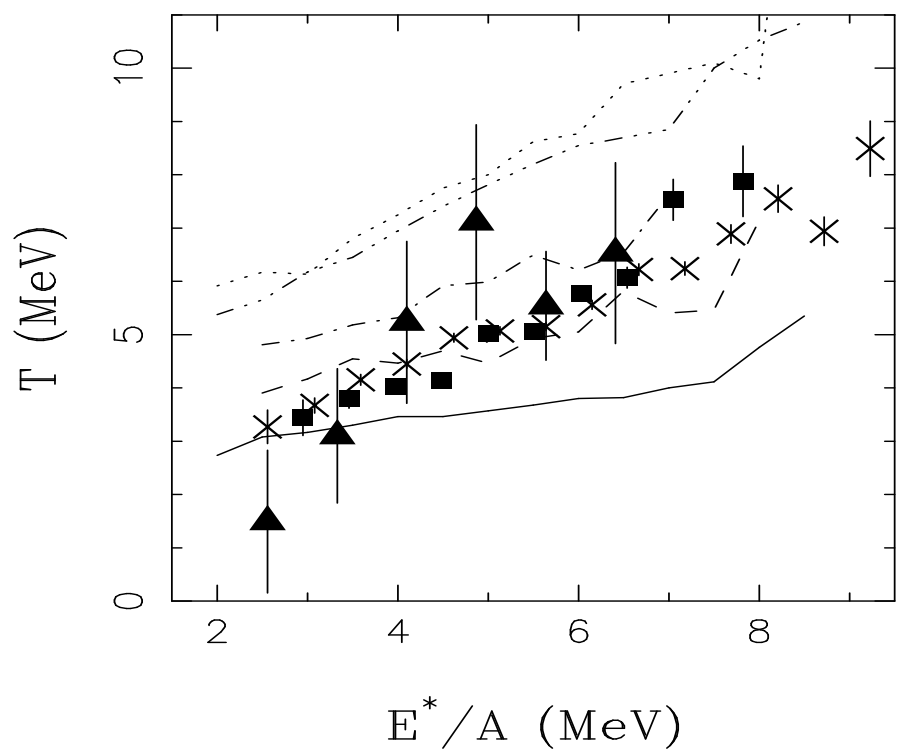

Figure 4: Caloric curve obtained using the double isotope ratio thermometer d,t $/{ }^{3,4} \mathrm{He}$ (squares), compared to the thermometer $\mathrm{d}, \mathrm{t} /{ }^{6,7} \mathrm{Li}$ (triangles), and to the kinematic temperatures of protons, deuterons, tritons, ${ }^{3} \mathrm{He}$ and alpha particles (solid, dashed, single dot-dashed, dotted and multiple dot-dashed lines, respectively). Combined fully resolved data with $\mathrm{Z} \geq 21$ from two reactions ${ }^{40,48} \mathrm{Ca}+{ }^{27} \mathrm{Al}$ at $45 \mathrm{MeV} /$ nucleon was used. Thin crosses show caloric curve obtained using the thermometer $\mathrm{d}, \mathrm{t} /{ }^{3,4} \mathrm{He}$ in the reactions ${ }^{28} \mathrm{Si}+{ }^{112,124} \mathrm{Sn}[12$.

As in the previous work [1, the isoscaling analysis was performed also for the reactions ${ }^{40,48} \mathrm{Ca}+{ }^{27} \mathrm{Al}$ at $45 \mathrm{MeV} /$ nucleon. Figure 3 shows isoscaling plots, plotted as a function of the projection of isospin, constructed using the identified fragments for the fully resolved quasiprojectiles with $Z \geq 21$ in six excitation energy bins. The observed isoscaling behavior is quite regular and the value of the isoscaling parameter (slope) decreases with excitation energy, in agreement with the previous experiment [1] and other experiments 
reported in the literature.
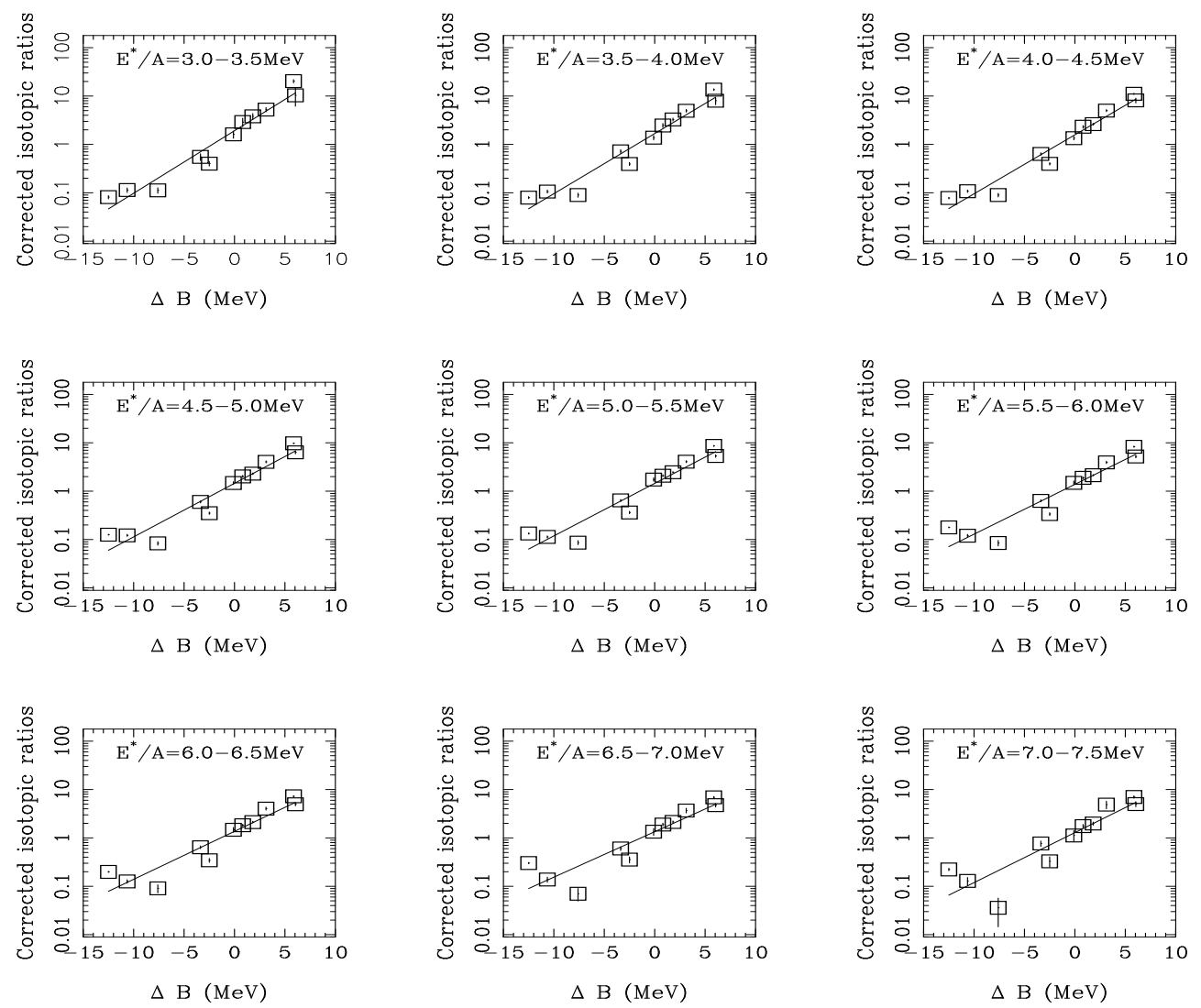

Figure 5: Isotopic yield ratios, corrected for the mass and for the ground state spin, plotted as a function of difference of binding energies for nine excitation energy bins.

In order to estimate the evolution of the chemical potential, as in the previous work [1], one needs to estimate the system temperature. Figure 4 shows the caloric curve obtained for the selected fully resolved data with $\mathrm{Z} \geq 21$ using the double isotope ratio thermometer $\mathrm{d}, \mathrm{t} / \mathrm{B}^{3,4} \mathrm{He}$ (squares). Furthermore, Figure 4 shows the comparison of the double isotope ratio thermometer $\mathrm{d}, \mathrm{t} /{ }^{3,4} \mathrm{He}$ to another thermometer $\mathrm{d}, \mathrm{t} /{ }^{6,7} \mathrm{Li}$ (triangles) and to the kinematic (slope) temperatures of protons, deuterons, tritons, ${ }^{3} \mathrm{He}$ and alpha particles. One can see that, unlike the previous work [13, there is no clear correspondence of any slope temperature to the double isotope ratio thermometers. Thus one needs to establish to what extent the double isotope 
ratio temperature of the two thermometers represents the multifragmentation temperature. A good judgment can be obtained when comparing the $\mathrm{d}, \mathrm{t} /{ }^{3,4} \mathrm{He}$ temperature observed in the present work to the earlier results for the reactions ${ }^{28} \mathrm{Si}+{ }^{112,124} \mathrm{Sn}[12,13]$. Such a comparison shows that the obtained caloric curves in both reactions are consistent and thus possibly represent general properties of the multifragmenting system. On the other hand, a similar comparison for the proton kinetic temperature shows that in the heavier system it dropped considerably, what can be explained by the onset of intense secondary emission of protons in the heavier system. Thus the mass range observed in the present work can represent an upper limit where the effect of secondary emission can be disentangled.

Moreover, since the double isotope ratio thermometers $\mathrm{d}, \mathrm{t} /{ }^{3,4} \mathrm{He}$ and $\mathrm{d}, \mathrm{t} /{ }^{6,7} \mathrm{Li}$ are just two of many possible thermometers, one can define a global temperature as an average value over the larger set of double isotope ratio thermometers. Such global temperature can be obtained using a graphical method developed in our previous works [22, 23]. The isotopic yield ratios, corrected for mass and ground state spin, can be plotted as a function of the difference of binding energies. The resulting plots for the present data are shown in Figure 5 for nine excitation energy bins.

The observed exponential (and thus again grand-canonical) scaling is reasonably good and one can assume that the fitted slope provides an global double isotope ratio temperature. Figure 6a shows the resulting caloric curve, along with the results of the double isotope ratio thermometer $\mathrm{d}, \mathrm{t} /{ }^{3,4} \mathrm{He}$ and the kinematic temperatures. It is remarkable to note, that there is a good correspondence between the global isotope ratio temperature and proton kinematic temperature. Both curves are relatively flat above $4 \mathrm{AMeV}$, which can be possibly interpreted as a long plateau. However, comparison with the lighter hot system in ref. [1] shows that the value of the plateau temperature is much lower than it can expected in multifragmentation. Especially in the case of thermometers using proton multiplicity and poton kinamatic temperature such low temperature with flat behavior can be caused by increased influence of the secondary de-excitation via nucleon (proton) emission, Such mode is not dominant in the lighter system,in ref. [1] where the Fermi decay, analogous to multifragmentation, dominates. Thus the proton kinematic temperature for the lighter system represents the earlier de-excitation stage, as is documented by higher values of the temperature [13] than in the present case.

The correspondence of the global isotope ratio temperature and the pro- 

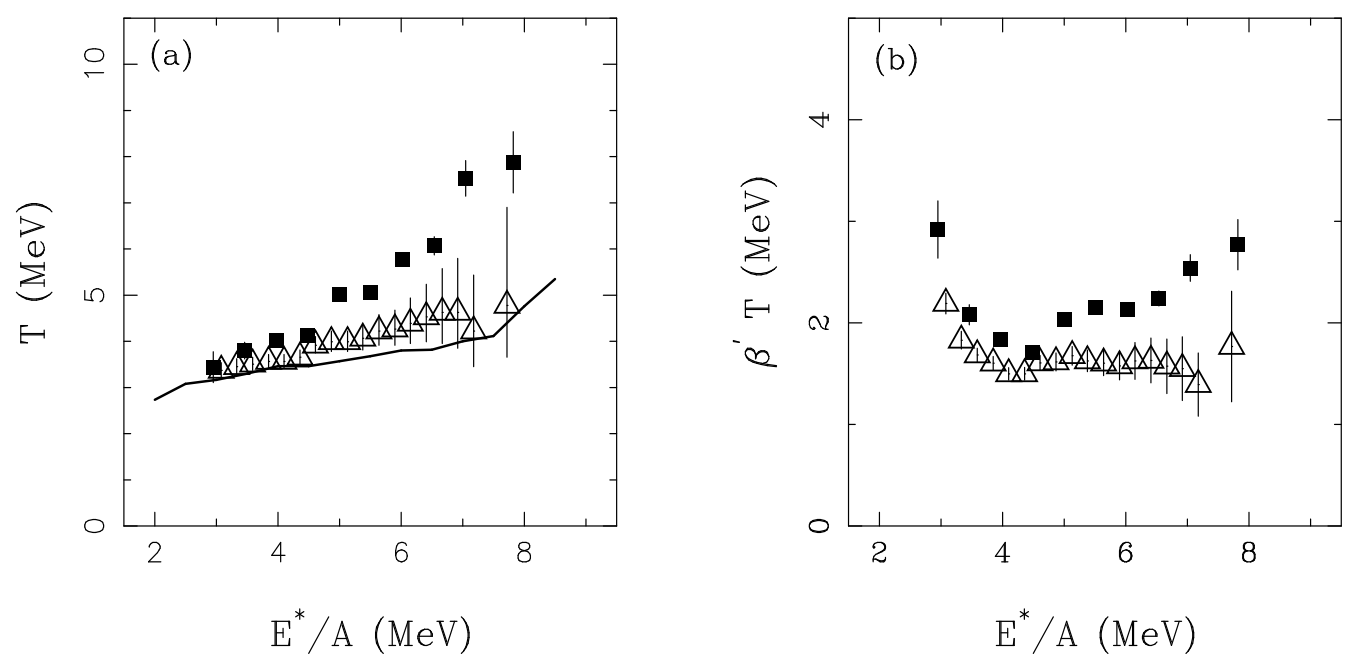

Figure 6: (a) Caloric curve obtained using global temperature from the slopes of $\Delta B$ dependences in Fig. 5 (triangles ) along with the caloric curves obtained using the double isotope ratio thermometer $\mathrm{d}, \mathrm{t} /{ }^{3,4} \mathrm{He}$ ( squares ) and proton slope temperatures ( line ). (b) Resulting values of $\beta \prime T$ obtained using the global ( triangles ) and $\mathrm{d}, \mathrm{t} /{ }^{3,4} \mathrm{He}$ ( squares ) temperatures.

ton kinematic temperature can thus be explained by secondary emission. The scaling behavior in Fig. [5 is determined mainly by isotopic ratios of intermediate mass fragments up to oxygen, where the nucleon (proton) emission is an increasingly dominant mode of secondary de-excitation. Thus, it is not surprising that identical temperatures are extracted from both the Maxwellian spectra and the fragment yield ratios. Unlike proton emission, the surface emission of the isotopes used in the $\mathrm{d}, \mathrm{t} /{ }^{3,4} \mathrm{He}$ thermometer is less probable. Therefore, their properties (as seen in their kinematic temperatures) reflect multifragmentation (volume emission) more closely. This is supported by the fact that the the isotopic ratio ${ }^{3} \mathrm{He} /{ }^{4} \mathrm{He}$ (first point from the left in each panel of Fig. 5) does not follow the systematics. The other isotopic ratio falling out of the systematics appears to be ${ }^{15} \mathrm{O} /{ }^{16} \mathrm{O}$ ( third from the left ). Its deviation from the systematics becomes larger with increasing excitation energy, and can be caused by an increasing influence of low lying excited states. A spin degeneracy factor of these excited states can be larger than the one of the ground state which was assumed in the correction. One thus ends up with the two distinct groups of thermometers. In the first group are the 
average isotope ratio temperature and proton kinematic temperature ( and also the double isotope ratio thermometers including protons ), representing the stage of secondary emission. The second group, the double isotope ratio thermometers $\mathrm{d}, \mathrm{t} /{ }^{3,4} \mathrm{He}$ and $\mathrm{d}, \mathrm{t} /{ }^{6,7} \mathrm{Li}$, appear to represent an earlier stage of de-excitation, i.e., multifragmentation of hot nuclei. The kinematic temperatures of $d, t,{ }^{3,4} \mathrm{He}$ also appear to be consistent with the earlier stage, as was the case in the previous work [13]. However, especially for deuterons and tritons one can not exclude admixture from the later stage, either by emission or by coalescence.

In macrocanonical limit, a product of the temperature $T$ and isoscaling parameter $\beta^{\prime}$ (from Fig. 3), corresponds to a value of the isovector part of chemical potential. The resulting values of $\beta^{\prime} T$ are shown in Figure $6 \mathrm{~b}$ for the $\mathrm{d}, \mathrm{t} /{ }^{3,4} \mathrm{He}$ thermometer (squares) and the average isotope ratio $(\Delta B)$ temperature (from Fig. 5, triangles). In the chemical potential corresponding to the $\mathrm{d}, \mathrm{t} /{ }^{3,4} \mathrm{He}$ thermometer, a reversion of the trend is observed at 4 $\mathrm{MeV} /$ nucleon, analogous to the results for lighter system [1]. Such behavior of chemical potential was also confirmed by lattice-gas calculations [24]. The estimate of the isovector chemical potential, obtained using the thermometer representing the secondary emission, still leads to approximately constant behavior above $4 \mathrm{MeV} /$ nucleon. It can be concluded that the increase of the isovector chemical potential, as documented for the $\mathrm{d}, \mathrm{t} /{ }^{3,4} \mathrm{He}$ thermometer, is related to de-excitation of the hot multifragmenting source and signals increasing isospin asymmetry of the gas phase in the isospin-asymmetric liquid-gas phase transition [1]. Furthermore, one needs to take into account that secondary emission influences also the value of the isoscaling parameter. The work in Ref. [25] showed that such influence is not significant for the lighter system ( $\mathrm{A} \simeq 25$ ), but for the systems with $\mathrm{A} \simeq 50$ ( as in the present case ) secondary emission and wide initial isotopic distributions from the dynamical stage of collision result in higher values of isoscaling parameters, and such trend weakens with increasing excitation energy. Thus the actual dependence can have an even deeper minimum since the effect of secondary emission tends to flatten it.

In general, one can conclude that the trends observed in the lighter quasiprojectiles in reactions ${ }^{28} \mathrm{Si}+{ }^{112,124} \mathrm{Sn}$ [1, 12, 13] were confirmed in reactions ${ }^{40,48} \mathrm{Ca}+{ }^{27} \mathrm{Al}$ at $45 \mathrm{MeV} /$ nucleon for the projectile-like nuclei with the mass about twice that of the former. Moreover, it has been shown that the properties of the hot systems, as reflected by the fragment observables, become still more distorted by secondary emission with the increasing mass. 


\section{Missing neutrons - statistical or dynamical emission ?}

Specifically for the neutron-rich projectile ${ }^{48} \mathrm{Ca}$, the effect of missing (undetected) neutrons must be considered. In the earlier works, it was established using successful simulations for the reactions ${ }^{28} \mathrm{Si}+{ }^{112,124} \mathrm{Sn}$ that the losses due to neutron emission represent a relatively small part of the system [10]. The isospin equilibration was not complete and thus the neutron-rich target did not result in a comparably neutron-rich quasi-projectile. The effect of missing neutrons was examined using isoscaling plots expressed for sets of identical quasi-projectiles from two reactions [1]. No dependence was observed which could be attributed to the influence of missing neutrons. It was also observed that the shape of the caloric curve did not depend on the $\mathrm{N} / \mathrm{Z}$ of the quasi-projectile.

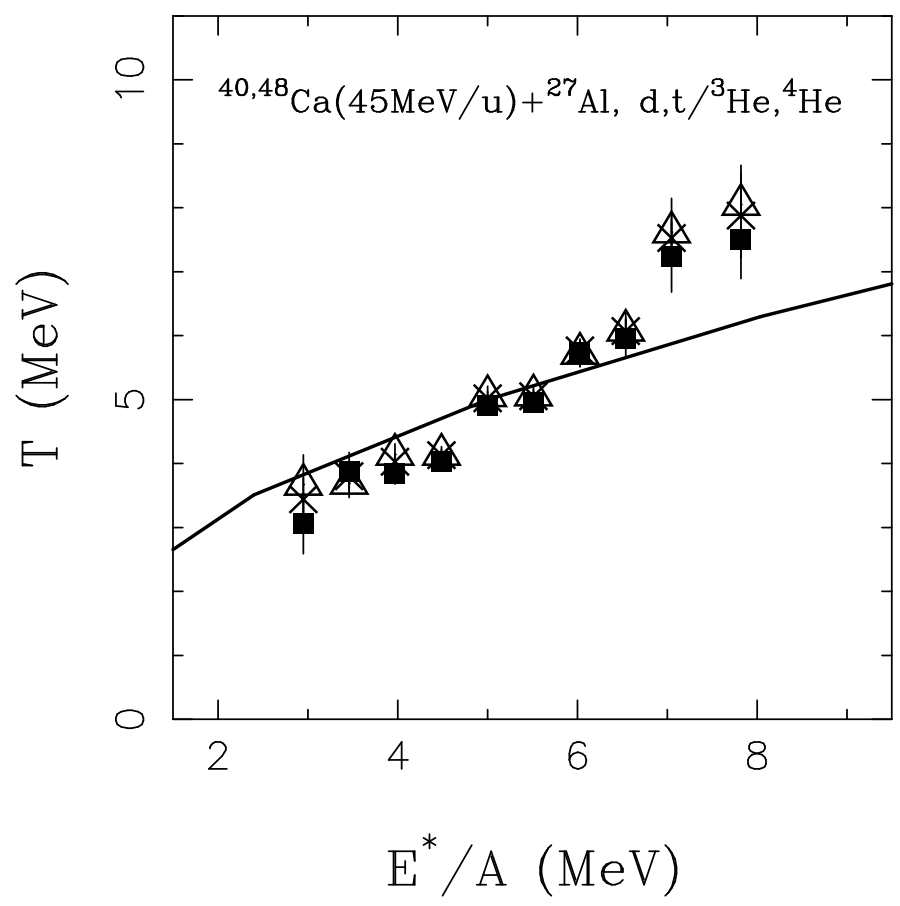

Figure 7: Caloric curves obtained using the double isotope ratio thermometer d,t $/{ }^{3,4} \mathrm{He}$ $\left({ }^{40} \mathrm{Ca}+{ }^{27} \mathrm{Al}\right.$ - squares, ${ }^{48} \mathrm{Ca}+{ }^{27} \mathrm{Al}$ - triangles, combined data - crosses, line - theoretical dependence from ref. [26] ).

In the case of the reactions ${ }^{40,48} \mathrm{Ca}+{ }^{27} \mathrm{Al}$, even when assuming an incomplete fusion scenario, one has to expect that there will be a considerable 
difference in neutron excess of the hot systems in the two reactions ( seven neutrons according to simulation ). In this context, it is remarkable that the caloric curves, expressed as a function of excitation energy obtained by charged particle calorimetry, are almost identical in both reactions, despite the expected significant difference of total excitation energy after including the missing neutrons. Figure 7 shows the caloric curves obtained using the double isotope ratio thermometer $\mathrm{d}, \mathrm{t} /{ }^{3,4} \mathrm{He}$ for the reactions ${ }^{40,48} \mathrm{Ca}+{ }^{27} \mathrm{Al}$, along with the theoretical line from ref. [26. There is a little difference observed between the two reactions. Especially, there is no shift which could be attributed to the difference of true excitation energies, which could exceed $1 \mathrm{MeV} /$ nucleon, due to a different number of emitted neutrons in the simulation ( up to seven ). The experimental caloric curves agree well with the theoretical dependence [26], representing temperatures where the isolated system with given excitation energy enters the spinodal contour, thus confirming the conclusion that, as in the reactions ${ }^{28} \mathrm{Si}+{ }^{112,124} \mathrm{Sn}$, the $\mathrm{d}, \mathrm{t} /{ }^{3,4} \mathrm{He}$ thermometer represents multifragmentation of the hot equilibrated source. Complementary to the caloric curves, the isoscaling behavior for selected N/Z-bins was also verified. The observed slopes, representing difference of chemical potentials, were consistent with zero, within statistical errors. Thermodynamical observables ( see Figs. 19, 2 and 7) thus, remarkably, do not exhibit sensitivity to the number of missing ( undetected ) neutrons in two reactions.

As an explanation one has to assume that the apparent excitation energy, reconstructed using observed charged particles, is close to the true excitation energy of the hot equilibrated source, and the excess neutrons in the neutronrich case are not part of the equilibrated source, due to dynamical emission taking place prior to equilibration. Such explanation is consistent with almost identical experimental excitation energy distributions shown in Fig. 1a for two reactions ${ }^{40,48} \mathrm{Ca}+{ }^{27} \mathrm{Al}$ and is also supported by the lack of the effect of missing neutrons on the scaling shown in Fig. 2 in the neutron-rich reaction ${ }^{48} \mathrm{Ca}+{ }^{27} \mathrm{Al}$. Dynamical emission of neutron-rich charged particles at mid-velocity was reported in recent years [27], and interpreted as caused by formation of a neutron-rich neck. Such scenario can provide an explanation also in the present case, with the notable difference that the neck structure may be formed exclusively by neutrons. In the present case the low-density region between the projectile and target can be expected to be very neutron-rich, since the nuclear equation of state for sub-saturation densities provides stable solutions only outside of the spinodal region and thus 
at very asymmetric $\mathrm{N} / \mathrm{Z}$ ratios. A behavior consistent with the present case was reported recently [28] in incomplete fusion reactions, where an isospindependent component of excitation energy was necessary to explain the production of neutron-rich fragmentation products. Such behavior is consistent with a rupture of the neck structure formed by the neutrons in the region between the hot and cold pre-fragment during the dynamical stage. Unlike the reported cases, where signatures of neck formation were reported in symmetric damped collisions of massive nuclei [27, in the present case the alternative explanation due to Coulomb force of the massive external charge [29] can be excluded. The remaining charge, especially in the incomplete fusion reactions, is rather small, and the observed trends can be attributed uniquely to the effect of the nuclear mean field.

\section{Summary and conclusions}

In summary, the signal of isospin-asymmetric phase transition in the evolution of chemical potential was observed in the multifragmentation of hot quasi-projectiles in the reactions ${ }^{40,48} \mathrm{Ca}+{ }^{27} \mathrm{Al}$ confirming an analogous observation in lighter quasi-projectiles in the reaction ${ }^{28} \mathrm{Si}+{ }^{12,124} \mathrm{Sn}$ [1]. However, with an increasing mass the properties of the hot systems become increasingly influenced by secondary emission. Thermodynamical observables exhibit no sensitivity to a different amount of missing neutrons due to variation of neutron excess of the hot system in the two reactions ${ }^{40,48} \mathrm{Ca}+{ }^{27} \mathrm{Al}$, thus providing a signal of dynamical emission of neutrons, which can be related to a formation of a neutron-rich low-density region (neck) between the projectile and target.

The authors wish to thank the staff of the Texas A\&M Cyclotron facility for the excellent beam quality. This work was supported by the US Department of Energy under contract DE-FG03-93ER40773, by the Welch Foundation under contract A-1266, by the Slovak Scientific Grant Agency under contracts VEGA-2/5098/25 and VEGA-2/0073/08, by the Slovak Research and Development Agency under Contract No. SK-CN-00706 and through the Agreement of Scientific Cooperation between China and Slovakia by the Ministry of Sciences and Technology and the Major State Basic Research Development Program (973 program) under contract No. 2007CB815004. 


\section{References}

[1] M. Veselsky, G.A. Souliotis, S.J. Yennello, Phys. Rev. C 69 (2004) $031602(\mathrm{R})$.

[2] H. Müller, B.D. Serot, Phys. Rev. C 52 (1995) 2072.

[3] V.M. Kolomietz et al., Phys. Rev. C 64 (2001) 024315.

[4] B.-A. Li, C. Ko, Nucl. Phys. A 618 (1997) 498; V. Baran et al., Phys. Rev. Lett. 86 (2001) 4492; M. Colonna et al., Phys. Rev. Lett. 88 (2002) 122701; J. Margueron, P. Chomaz, Phys. Rev. C 67 (2003) 041602(R).

[5] S.J. Yennello et al., Phys. Lett. B 321 (1994) 14.

[6] H. Johnston et al., Phys. Lett. B 371 (1996) 186.

[7] E. Ramakrishnan et al., Phys. Rev. C 57 (1998) 1803.

[8] R. Laforest et al., Phys. Rev. C 59 (1999) 2567.

[9] H.S. Xu et al., Phys. Rev. Lett. 85 (2000) 716.

[10] M. Veselsky et al., Phys. Rev. C 62 (2000) 064613.

[11] M. Veselsky et al., Phys. Rev. C 62 (2000) 041605(R).

[12] M. Veselsky et al., Phys. Lett. B 497 (2001) 1.

[13] M. Veselsky and S.J. Yennello, Nucl. Phys. A 749 (2005) 114c.

[14] L. Tassan-Got, C. Stéfan, Nucl. Phys. A 524 (1991) 121.

[15] J.P. Bondorf et al., Phys. Rep. 257 (1995) 133.

[16] F. Gimeno-Nogues et al., Nucl. Instrum. and Meth. A 399 (1997) 94.

[17] M. Veselsky et al., Progress in Research, 2000-2001, Cyclotron Institute, Texas A\&M University, College Station, 2001. P.V-13.

[18] L. Tassan-Got, Nucl. Instrum. Meth. B 194 (2002) 503.

[19] M.B. Tsang et al., Phys. Rev. Lett. 86 (2001) 5023. 
[20] M. Veselsky, Nucl. Phys. A 705 (2002) 193.

[21] M. Veselsky et al., Nucl. Phys. A 724 (2003) 431.

[22] M. Veselsky, G.A. Souliotis, S.J. Yennello, Proc. 5th Int. Conf. DANF2001, Casta-Papiernicka, Slovakia, 2001, World Scientific, 2002. P.461. E-print arXiv.org:nucl-ex/0112007.

[23] D.V. Shetty et al., Phys. Rev. C 68 (2003) 054605.

[24] Y.G. Ma et al., Physical Review C 69 (2004) 064610.

[25] M. Veselsky, Phys.Rev. C 74 (2006) 054611.

[26] M. Veselsky, E-print arXiv.org:nucl-th/0703077.

[27] G. Casini et al., Phys. Rev. Lett. 71 (1993) 2567; C.P. Montoya et al., Phys. Rev. Lett. 73 (1994) 3070; J.F. Dempsey et al., Phys. Rev. C 54 (1996) 1710.

[28] M. Veselsky, G.A. Souliotis, Nucl. Phys. A 781 (2007) 521.

[29] M. Jandel et al., Journal of Phys. G 31 (2005) 29. 